Claremont Colleges

Scholarship@ Claremont

All HMC Faculty Publications and Research

HMC Faculty Scholarship

8-1-1988

\title{
Multiple Solutions for a Dirichlet Problem with Jumping Nonlinearities II
}

Alfonso Castro

Harvey Mudd College

Ratnasingham Shivaji

Mississippi State University

\section{Recommended Citation}

Castro, Alfonso and R. Shivaji. "Multiple solutions for a Dirichlet problem with jumping nonlinearities II," Journal of Math. Analysis and Appl. Vol. 133, No. 2 (1988) 509-528.

This Article - postprint is brought to you for free and open access by the HMC Faculty Scholarship at Scholarship @ Claremont. It has been accepted for inclusion in All HMC Faculty Publications and Research by an authorized administrator of Scholarship @ Claremont. For more information, please contact scholarship@cuc.claremont.edu. 


\title{
Multiple Solutions for a Dirichlet Problem with Jumping Nonlinearities, II
}

\author{
Alfonso Castro \\ Department of Mathematics, North Texas State University, \\ Denton, Texas 76203-5116 \\ AND \\ R. SHIVAJI \\ Department of Mathematics, Mississippi State University, \\ Mississippi State, Mississippi 39762 \\ Submitted by V. Lakshmikantham \\ Received March 20, 1986
}

\section{INTRODUCTION}

In this paper we consider the existence of solutions for the problem

$$
\begin{aligned}
-x^{\prime \prime}(t) & =g(x(t))-p(t)-c, \quad t \in[0,1] \\
x(0) & =x(1)=0,
\end{aligned}
$$

where $p(t)$ is a continuous function and $c$ is a constant. We assume that $g$ is of class $C^{1}$, and that $g$ is positive and strictly increasing on $(0, \infty)$. In addition, we assume that there exist real numbers $M$ and $\rho>0$ such that

$$
\begin{array}{r}
\lim _{u \rightarrow-\infty}(g(u) / u)=M, \\
\lim _{u \rightarrow \infty}\left(g(u) / u^{1+\rho}\right)=\infty .
\end{array}
$$

Our main result is:

THEOREM 1.1. If $p$ and $g$ are as above, then there exists an increasing sequence $\left\{c(n):=c(n, p) ; n>2(\max \{0, M\})^{1 / 2} / \pi\right\}$ tending to $+\infty$ such that

(A) If $n>1+2(\max \{0, M\})^{1 / 2} / \pi$ and $c>c(n)$ then (1.1)-(1.2) have a solution with $n$ interior zeroes and $x^{\prime}(0)>0$.

(B) If $n>2\left(\max \{0, M\}^{1 / 2}\right) / \pi$ and $c>c(n)$ then (1.1)-(1.2) have a solution with $n$ interior zeroes and $x^{\prime}(0)<0$. 
Further if $g(u)-\pi^{2} u$ is bounded below then there exists $c_{*}$ such that for $c<c_{*}$ Eqs. (1.1)-(1.2) have no solution.

Our proofs are based on the analysis of the phase-plane. Most of our arguments resemble those of [2], where under the hypothesis $\lim _{|u| \rightarrow \infty}(g(u) / u)=\infty$ the existence of infinitely many solutions for (1.1)-(1.2) was proved. We do not make use of the maximum principle. This is why, unlike in previous work (see $[1,5]$ and references therein), we are not restricted to $M<\lambda_{1}=\pi^{2}$.

The outline of the proof of Theorem 1.1 is as follows. In the first place show that there exist constants $\alpha \in(0,1)$ and $c^{*}$ such that if $c>c^{*}$ and $|a| \geqslant 1$ then the solution to Eq. (1.1) satisfying $x(0)=0, x^{\prime}(0)=a c^{\alpha}$ exists on $[0,2]$ (see Lemma 2.1 ) and that its zeroes on $[0,2]$ are non-degenerate (see Lemma 2.2). That is, the orbit starting at $\left(0, a c^{x}\right)$ does not go through the origin in the $\left(x, x^{\prime}\right)$-plane for $t \in[0,2]$. Thus for such $(t, a, c)$ a continuous argument function $\theta(t, a, c)$ is well defined (see [4]). Next we show that if $|a| \in[1,3]$ then $\lim _{c \rightarrow \infty} \theta(1, a, c)=\infty$ (see Lemmas 2.3 and 2.4) and for $c>c^{*}, \lim _{|a| \rightarrow \infty} \theta(1, a, c) \leqslant \pi+2(\max \{0, M\})^{1 / 2}$ (see Lemmas 2.5 and 2.6). Using these two limits and the intermediate value theorem we conclude that given suitably large $n$ (see Theorem 1.1) there exists $c(n)$ such that if $c>c(n)$ then for some $a, \theta(1, a, c)=(n+1) \pi$, which by the definition of $\theta$ implies that the corresponding orbit is a solution to (1.1) (1.2) with $n$ interior zeroes.

Theorem 1.1 extends the work of [3], where the case $p=0$ was studied using the so-called quadrature method. Our motivation to study problem (1.1) $-(1.2)$ is due to the results of [5], where $M<\pi^{2}$ and $\lim _{u \rightarrow \infty}(g(u) / u) \in\left(N^{2} \pi^{2},(N+1)^{2} \pi^{2}\right)$. In turn, A. C. Lazer and P. J. McKenna in [5] were motivated by developments that go back to the classical Ambrosetti-Prodi result [1].

\section{Phase-Plane Analysis}

In what follows we extend $p$ to $[0, \infty)$ as $p(x)=p(1)$ for $x \geqslant 1$. Also, without loss of generality, we can assume that

$$
\max \{|p(x)| ; x \geqslant 0\} \leqslant 1 \quad \text { and } \quad g(0)=0 .
$$

LEMMa 2.1. If $a, b, c$ are arbitrary numbers then the solution to Eq. (1.1) satisfying $x(0)=a, x^{\prime}(0)=b$ exists for all $t$.

Proof. Since $g$ is of class $C^{1}$, by (1.3), (1.4), and (2.1), we see that there exists a real number $M_{1} \geqslant 0$ such that $g_{1}(u):=g(u)+M_{1} u$ satisfies

$$
u g_{1}(u) \geqslant 2 u^{2} \quad \text { for all } u \in R \text {. }
$$


Let $G_{1}(u)=\int_{0}^{u} g_{1}(s) d s$ and

$$
E_{1}(t)=\left(x^{\prime}(t)\right)^{2} / 2+G_{1}(x(t))
$$

where $x(t)$ is the solution to (1.1) satisfying $x(0)=a, x^{\prime}(0)=b$. Hence

$$
\begin{aligned}
\frac{d E_{1}}{d t} & =\left(x^{\prime}(t)\right)\left[x^{\prime \prime}(t)+g_{1}(x(t))\right] \\
& =x^{\prime}(t)\left[-g(x(t))+p(t)+c+g(x(t))+M_{1} x(t)\right] \\
& \leqslant|c+1| \sqrt{2 E_{1}}+M_{1} \sqrt{2 E_{1}}|x(t)|,
\end{aligned}
$$

where we have used that $G_{1} \geqslant 0$ (see (2.2)). Also from (2.2) we have $G_{1}(u) \geqslant u^{2}$ for all $u \in R$. This, (2.3), and the fact that $E_{1} \geqslant 0$ yield

$$
\begin{aligned}
\frac{d E_{1}}{d t} & \leqslant|c+1| \sqrt{2 E_{1}}+M_{1} \sqrt{2 E_{1}} \cdot \sqrt{E_{1}} \\
& \leqslant \sqrt{2}|c+1|+\left(|c+1|+M_{1} \sqrt{2}\right) E_{1} .
\end{aligned}
$$

Hence if we let $k=-\left(|c+1|+\sqrt{2} M_{1}\right)$, then multiplying (2.4) by $e^{k t}$ we have

$$
\frac{d}{d t}\left(e^{k t} E_{1}\right) \leqslant e^{k t} \sqrt{2}|c+1|
$$

Thus we have

$$
e^{k t} E_{1}(t)-E_{1}(0) \leqslant \int_{0}^{u} e^{k s} \sqrt{2}|c+1| d s
$$

i.e.,

$$
e^{k t} E_{1}(t) \leqslant \frac{b^{2}}{2}+G_{1}(a)+\frac{\sqrt{2}|c+1| e^{k t}}{k}-\frac{\sqrt{2}|c+1|}{k},
$$

i.e.,

$$
E_{1}(t) \leqslant e^{-k t}\left\{\frac{b^{2}}{2}+G_{1}(a)-\frac{\sqrt{2}|c+1|}{k}\right\}+\frac{\sqrt{2}|c+1|}{k} .
$$

Therefore $E_{1}$ is bounded on bounded intervals. Hence, since $G_{1}(u) \geqslant u^{2}$ (see (2.2)), $\left(x, x^{\prime}\right)$ is bounded on bounded intervals. This proves that $\left(x, x^{\prime}\right)$ does not blow up in finite time, which proves that $\left(x, x^{\prime}\right)$ is defined for all time and the lemma is proven.

Now let $\alpha$ be defined by

$$
\alpha=(\rho+3) /(2 \rho+3) .
$$


Let $x(t, \alpha)$ denote the solution to (1.1) satisfying $x(0)=0, x^{\prime}(0)=a c^{\alpha}$ with $a \in R$. Let $r^{2}(t, a)=(x(t, a))^{2}+\left(x^{\prime}(t, a)\right)^{2}$. Now for $a \in[1, \infty)$ and for $T>0$ such that $r(t, a)>0$ on $[0, T]$, there exists the function $\theta(t, a)$ such that

$$
\begin{aligned}
x(t, a) & =r(t, a) \sin (\theta(t, a)), \\
x^{\prime}(t, a) & =r(t, a) \cos (\theta(t, a)), \\
\theta(0, a) & =0 .
\end{aligned}
$$

A simple computation shows that

$$
\begin{aligned}
\theta^{\prime}(t, a)= & \frac{\left(x^{\prime}(t, a)\right)^{2}-x(t, a) x^{\prime \prime}(t, a)}{r^{2}(t, a)} \\
= & \left(\cos ^{2}(\theta(t, a))\right)+\frac{g(r(t, a) \sin (\theta(t, a))) \sin (\theta(t, a))}{r(t, a)} \\
& -(p(t)+c) \frac{\sin (\theta(t, a))}{r(t, a)} .
\end{aligned}
$$

Since the dependence of the various functions on $a$ is clear from the context we will eventually drop this variable.

LEMmA 2.2. There exists $c^{*}$ such that if $c>c^{*}$ and $|a| \geqslant 1$ then $r(t, a)>0$ for all $t \in[0,2]$.

Proof. Let $M_{1}, G_{1}$, and $E_{1}$ be as before. Suppose $E_{1}\left(t^{\prime}, a\right)=0$ for some $t^{\prime} \in(0,2]$ and $E_{1}>0$ for $t \in\left[0, t^{\prime}\right)$. By the continuity of $E_{1}$ we can assume that there exists $t^{\prime \prime}<t^{\prime}$ such that $E_{1}\left(t^{\prime \prime}, a\right)=c^{2 \alpha} / 2$ and $0<E_{1}(t, a)<c^{2 \alpha} / 2$ for all $t \in\left(t^{\prime \prime}, t^{\prime}\right)$. Then, since $G_{1} \geqslant 0$, we have $\left|x^{\prime}\right|<c^{\alpha}$ on $\left(t^{\prime \prime}, t^{\prime}\right)$. Also from (1.4) we obtain

$$
x\left(t^{\prime \prime}\right)<K c^{2 x /(2+\rho)},
$$

with $K>0$ a constant independent of $\mathbf{c}$ and a. Now since $d E_{1} / d t=$ $\left(c+p(t)+M_{1} x(t)\right) x^{\prime}(t)$, integrating in $\left(t^{\prime \prime}, t^{\prime}\right)$ we obtain

$$
\begin{aligned}
0=E_{1}\left(t^{\prime}\right) & =E_{1}\left(t^{\prime \prime}\right)+\int_{t^{\prime \prime}}^{t^{\prime}}\left(c+p(s)+M_{1} x(s)\right) x^{\prime}(s) d s \\
& \geqslant \frac{c^{2 \alpha}}{2}-c x\left(t^{\prime \prime}\right)-2 c^{\alpha}-\frac{M_{1}}{2} x^{2}\left(t^{\prime \prime}\right) \\
& >\frac{c^{2 \alpha}}{2}-K c^{1+(2 \alpha /(2+\rho))}-2 c^{\alpha}-\frac{M_{1} K^{2}}{2} c^{4 \alpha /(2+\rho)} .
\end{aligned}
$$


But by the definition of $\alpha$ (see (2.7)), $2 \alpha>\max \{1+2 \alpha /(2+\rho), 4 \alpha /(2+\rho)\}$ and hence (2.14) is not possible for $c$ large. That is, there exists $c^{*}$ such that for $c>c^{*}, E_{1}(t, a)>0$ for all $t \in[0,2]$. Hence $r(t, a)>0$ if $|a| \geqslant 1$, $t \in[0,2]$, and $c>c^{*}$, which proves Lemma 2.2.

Lemma 2.3. Let $a_{1} \in[1,3], T \in[0,1]$, and $K$ be a non-negative integer. Given any positive integer $n$ there exists $d=d(n)$ such that if $c>d$, $\theta\left(T, a_{1}\right)=2 K \pi$, and $x^{\prime}\left(T, a_{1}\right)=a c^{\alpha}$ with $a \in[1,3]$, then there exists $T_{1} \in[0,1 / n]$ such that $\theta\left(T+T_{1}, a_{1}\right)=(2 K+1) \pi$. Moreover

$$
\left|x^{\prime}\left(T+T_{1}, a_{1}\right)+a c^{\alpha}\right|<c^{\alpha} /\left(2 n^{2}\right) .
$$

Proof. For the sake of clarity we divide this proof in three steps. Throughout this proof $\varepsilon$ denotes a fixed rcal number satisfying

$$
0<\varepsilon<(1-\alpha) / 2
$$

Step 1. There exists $d_{1}$ such that if $c>d_{1}$ then there exists $t_{1} \leqslant$ $T+\left(c^{-\varepsilon} / 2\right)$ such that $x\left(t_{1}, a_{1}\right)=g^{-1}\left(c-1+c^{\alpha+\varepsilon}\right)$ and $x^{\prime}\left(t_{1}\right)>0$.

First note that by $(2.16), \alpha+\varepsilon=(\alpha+1) / 2<1$ since $\alpha<1$. Further by (2.7) and (2.16) we have $1 /(\rho+1)+\varepsilon-\alpha<0$. These and (1.4) imply

$$
\lim _{c \rightarrow \infty}\left\{c^{\varepsilon}\left[g^{-1}\left(c-1+c^{\alpha+\varepsilon}\right)\right] / c^{\alpha}\right\}=0 .
$$

Further, by (2.7) we have $1+1 /(\rho+1)-2 \alpha<0$. Hence

$$
\lim _{c \rightarrow \infty}\left\{(c+1)\left[g^{-1}\left(c-1+c^{\alpha+\varepsilon}\right)\right] / c^{2 \alpha}\right\}=0 .
$$

From (2.17) it follows that there exists $\delta_{1}>0$ such that if $c>\delta_{1}$ then

$$
\cos ^{2}\left(\arctan \left[g^{-1}\left(c-1+c^{\alpha+\varepsilon}\right) / c^{\alpha}\right]\right)>0.95 \text {. }
$$

Since the derivative with respect to $z$ of $\sin (\arctan (z))$ at $z=0$ is 1 , by (2.18) we see that there exists $\delta_{2}>0$ such that if $c>\delta_{2}$ then

$$
(c+1) \sin \left[\arctan \left(2 g^{-1}\left(c-1+c^{\alpha+\varepsilon}\right) / c^{\alpha}\right)\right] /\left(c^{\alpha} / 2\right)<0.05 .
$$

Again by (2.17) we see that there exists $\delta_{3}>0$ such that if $c>\delta_{3}$ then

$$
g^{-1}\left(c-1+c^{\alpha+\varepsilon}\right) /\left(a c^{\alpha}\right)<0.1 c^{-\varepsilon}
$$

and

$$
\arctan \left[2 g^{-1}\left(c-1+c^{\alpha+\varepsilon}\right) /\left(a c^{\alpha}\right)\right] \leqslant 3 g^{-1}\left(c-1+c^{\alpha+\varepsilon}\right) /\left(a c^{\alpha}\right) .
$$


Let $d_{1}$ be defined by

$$
d_{1}=\max \left\{\delta_{1}, \delta_{2}, \delta_{3}, c^{*}, 2\right\},
$$

where $c^{*}$ is as in Lemma 2.2 .

Let $c>d_{1}$. Since by hypothesis $\theta\left(T, a_{1}\right)=2 K \pi$, by (2.8) we have $x\left(T, a_{1}\right)=0$. This and the assumption $x^{\prime}\left(T, a_{1}\right)=a c^{x}>0$ imply that there exists $t>T$ such that $x(s) \in\left(0, g^{-1}\left(c-1+c^{x+\varepsilon}\right)\right)$ for all $s \in(T, t)$. Thus the set $S=\left\{t>T ; x(s) \in\left(0, g^{-1}\left(c-1+c^{x+v}\right)\right)\right\}$ is non-empty. Therefore we have the following alternatives. Either

(A) The interval $\left[T, T+\left(c^{-\varepsilon} / 2\right)\right] \subset S$, or

(B) There exists $t \in S, \quad t \leqslant T+\left(c^{-\varepsilon} / 2\right)$ such that $x(t)=$ $g^{-1}\left(c-1+c^{\alpha+\varepsilon}\right)$, or

(C) There exists $t \in S, t \leqslant T+\left(c^{-\varepsilon} / 2\right)$ such that $x(t)=0$.

Now we will show that neither (A) nor (C) is possible. Let $t \in S$ with $t \leqslant T+\left(c^{-\varepsilon} / 2\right)$. Integrating $(1.1)$ on $(T, t)$ we have

$$
\begin{aligned}
x^{\prime}(t) & =a c^{\alpha}+\int_{T}^{t}(p(\tau)+c-g(x(\tau))) d \tau \\
& \geqslant a c^{\alpha}+(c-1)(t-T)-\left(c-1+c^{\alpha+\varepsilon}\right)(t-T)
\end{aligned}
$$

since $x(\tau) \leqslant g^{-1}\left(c-1+c^{\alpha+\varepsilon}\right)$ and $g(x)$ is increasing in $(0, \infty)$. But $t-T \leqslant c^{-\varepsilon} / 2$. Hence we have

$$
x^{\prime}(t) \geqslant a c^{\alpha}-\left(c^{\alpha} / 2\right) \geqslant a c^{\alpha} / 2 .
$$

Thus by the definition of $r(t, a)$ we have $r\left(s, a_{1}\right) \geqslant a c^{\alpha} / 2$ for all $s \in[T, t)$. Now since $\theta\left(s, a_{1}\right)=\arctan \left(x / x^{\prime}\right)$ we get

$$
\theta\left(s, a_{1}\right) \leqslant \arctan \left[2 g^{-1}\left(c-1+c^{\alpha+\varepsilon}\right) /\left(a c^{\alpha}\right)\right]
$$

for all $s \in[T, t)$. Replacing this in (2.20) we have

$$
(c+1) \sin \left(\theta\left(s, a_{1}\right)\right) / r\left(s, a_{1}\right) \leqslant 0.05
$$

for $c>d_{1}$ and $s \in[T, t)$. Hence from (2.12), (2.19), and (2.26) we have

$$
\theta^{\prime}\left(s, a_{1}\right) \geqslant 0.9
$$

for all $s \in[T, t)$. Thus if (A) holds then

$$
\theta\left(T+\left(c^{-\varepsilon} / 2\right), a_{1}\right) \geqslant 0.45 c^{-\varepsilon} .
$$


This, (2.25), (2.22), and (2.21) give

$$
\begin{aligned}
0.45 c^{-\varepsilon} & \leqslant \arctan \left[2 g^{-1}\left(c-1+c^{\alpha+\varepsilon}\right) /\left(a c^{\alpha}\right)\right] \\
& \leqslant 3 g^{-1}\left(c-1+c^{\alpha+\varepsilon}\right) /\left(a c^{\alpha}\right) \\
& \leqslant 0.3 c^{-\varepsilon}
\end{aligned}
$$

which is clearly false. This contradiction shows that (A) cannot occur. On the other hand, if (C) occurs then by the definition of the set $S$ we have $x(s)>0$ for all $s \in(T, t)$. Therefore

$$
x^{\prime}(t) \leqslant 0
$$

Since (2.29) contradicts (2.24), we see that (C) cannot occur either. Thus only (B) holds, and by $(2.24), x^{\prime}\left(t_{1}\right) \geqslant a c^{\alpha} / 2>0$. Hence Step 1 holds.

Step 2. Let $t_{1}$ and $d_{1}$ be as in Step 1. We claim that there exists $d_{2} \geqslant d_{1}$ such that if $c>d_{2}$ then for some $t_{2} \in\left(t_{1}, t_{1}+\left[2 a c^{\alpha} /\left(c^{\alpha+\varepsilon}-2\right)\right]\right)$ we have $x\left(t_{2}\right)=g^{-1}\left(c-1+c^{\alpha+\varepsilon}\right), \quad x^{\prime}\left(t_{2}\right)<0, \quad$ and $x(s)>g^{-1}\left(c-1+c^{\alpha+\varepsilon}\right)$ for $s \in\left(t_{1}, t_{2}\right)$.

By Step 1 (in particular see (2.24))

$$
x^{\prime}\left(t_{1}\right) \geqslant\left(a c^{\alpha} / 2\right)>0 \text {. }
$$

Therefore there exists $t>t_{1}$ such that $x(s)>g^{-1}\left(c-1+c^{\alpha+\varepsilon}\right)$ for $s \in\left(t_{1}, t\right)$. Integrating (1.1) on $\left[t_{1}, t\right]$ we have

$$
\begin{aligned}
x^{\prime}(t) & =x^{\prime}\left(t_{1}\right)+\int_{t_{1}}^{t}(c+p(\tau)-g(x(\tau))) d \tau \\
& \leqslant x^{\prime}\left(t_{1}\right)+\left[c+1-\left(c-1+c^{\alpha+\varepsilon}\right)\right]\left(t-t_{1}\right) \\
& \leqslant x^{\prime}\left(t_{1}\right)+\left(2 \cdot c^{\alpha+\varepsilon}\right)\left(t \quad t_{1}\right) .
\end{aligned}
$$

Since, by hypothesis, $g$ is an increasing function on $[0, \infty)$ and $x$ is also an increasing function on $\left[T, t_{1}\right)$ (see $(2.24)$ ), we have $c+p(s)-g(x(s)) \leqslant 0$ whenever $x(s) \geqslant g^{-1}(c+1)$ and $s \in\left[T, t_{1}\right)$. Therefore by (1.1) we have

$$
x^{\prime}\left(t_{1}\right) \leqslant x^{\prime}\left(s_{1}\right)
$$

where $s_{1} \in\left[T, t_{1}\right)$ is such that

$$
x\left(s_{1}\right)=g^{-1}(c+1) .
$$

The existence of such an $s_{1}$ follows for $c$ larger than $2^{1 / \alpha}$ and by the continuity of $x$. 
Again using (2.24) we have

$$
\begin{aligned}
g^{\prime}(c+1) & =0+\int_{T}^{s_{1}} x^{\prime}(s) d s \\
& \geqslant\left(s_{1}-T\right)\left(a c^{x} / 2\right) .
\end{aligned}
$$

Next we observe that there is a $\delta_{4} \geqslant d_{1}$ such that if $c \geqslant \delta_{4}$ then

$$
\begin{aligned}
g^{-1}(c+1) & \leqslant c^{1 /(\rho+1)}, \\
2 c^{-\varepsilon} & <c^{\alpha},
\end{aligned}
$$

and

$$
2(c+1) c^{(1 /(\rho+1))-\alpha}<c^{\alpha} .
$$

Inequality (2.35) follows immediately from (1.4), whereas (2.36) follows from the hypotheses $\varepsilon>0$ and $\alpha>0$. By (2.7) we have $1+(1 /(\rho+1))<2 \alpha$, which implies (2.37). Thus we have established the existence of $\delta_{4}$ satisfying (2.35)-(2.37).

Let $c>d_{2}=\operatorname{Max}\left\{\delta_{4}, 2^{1 / \alpha}\right\}$. Then replacing (2.35) into (2.34) we have

$$
\left(s_{1}-T\right) \leqslant(2 / a) c^{(1 /(\rho+1))-\alpha}
$$

for all $c \geqslant d_{2}$. Hence integrating $(1.1)$ on $\left[T, s_{1}\right]$ we obtain

$$
\begin{aligned}
x^{\prime}\left(s_{1}\right) & =x^{\prime}(T)+\int_{T}^{s_{1}}(c+p(\tau)-g(x(\tau))) d \tau \\
& \leqslant a c^{\alpha}+(c+1)\left(s_{1}-T\right) \\
& \leqslant a c^{\alpha}+(c+1)(2 / a) c^{(1 /(\rho+1))-\alpha} .
\end{aligned}
$$

Now replacing (2.37) in the last term of (2.39) and using (2.32) we arrive at

$$
x^{\prime}\left(t_{1}\right) \leqslant a c^{\alpha}+c^{\alpha} / a \leqslant 2 a c^{\alpha}
$$

since $a \geqslant 1$.

Suppose now that $t>t_{1}$ and $x(s)>g^{-1}\left(c-1+c^{\alpha+\varepsilon}\right)$ for all $s \in\left(t_{1}, t\right)$. From (2.31) and (2.40) we have

$$
x(t)-x\left(t_{1}\right) \leqslant 2 a c^{\alpha}\left(t-t_{1}\right)+\left(2-c^{\alpha+\varepsilon}\right)\left(t-t_{1}\right)^{2} .
$$

Hence if $t>t_{1}+\left[2 a c^{\alpha} /\left(c^{\alpha+\varepsilon}-2\right)\right]$ we infer

$$
x(t)-x\left(t_{1}\right)<0 \text {. }
$$


This and the continuity of $x$ imply that there exists $t_{2} \in\left(t_{1}, t_{1}+\right.$ $\left.\left[2 a c^{\alpha} /\left(c^{\alpha+\varepsilon}-2\right)\right]\right)$ such that $x\left(t_{2}\right)=x\left(t_{1}\right)=g^{-1}\left(c-1+c^{\alpha+\varepsilon}\right)$ and $x(s)>$ $g^{-1}\left(c-1+c^{\alpha+\varepsilon}\right)$ for all $s \in\left(t_{1}, t_{2}\right)$. Clearly $x^{\prime}\left(t_{2}\right) \leqslant 0$. We will now show that, in fact, $x^{\prime}\left(t_{2}\right)<0$. Since $x(s)>g^{-1}\left(c-1+c^{\alpha+\varepsilon}\right)$ for $s \in\left(t_{1}, t_{2}\right)$, by (1.1) we have

$$
\begin{aligned}
x^{\prime \prime}(s) & <(c+1)-\left(c-1+c^{\alpha+\varepsilon}\right) \\
& =2-c^{\alpha+\varepsilon}<0
\end{aligned}
$$

for all $s \in\left(t_{1}, t_{2}\right)$, since $c>d_{2} \geqslant 2^{1 / x}$. This and the fact that $x\left(t_{1}\right)=x\left(t_{2}\right)=$ $g^{-1}\left(c-1+c^{\alpha+\varepsilon}\right)$ imply that there exists a unique $s_{2} \in\left(t_{1}, t_{2}\right)$ with $x\left(t_{2}\right)=$ $\operatorname{Max}\left\{x(s) ; t_{1} \leqslant s \leqslant t_{2}\right\}$ and $x^{\prime}\left(s_{2}\right)=0$.

Thus, using again that $x^{\prime \prime}(s)<0$ on $\left(t_{1}, t_{2}\right)$, we have

$$
x^{\prime}\left(t_{2}\right)<x^{\prime}\left(s_{2}\right)=0 \text {. }
$$

Since also $x\left(t_{2}\right)=g^{-1}\left(c-1+c^{\alpha+\varepsilon}\right)$, and $x(s)>g^{-1}\left(c-1+c^{\alpha+\varepsilon}\right)$, for all $s \in\left(t_{1}, t_{2}\right)$ Step 2 is proven.

Step 3. Let $t_{1}, t_{2}, d_{1}, d_{2}$ be as above. Then there exists $d_{3} \geqslant d_{2}$, $t_{3} \in\left(t_{2}, t_{2}+c^{\beta(\alpha-1)}\right]$, where

$$
1<\beta<1+1 /(\rho+1)
$$

such that if $c>d_{3}$ then

$$
x\left(t_{3}\right)=0 \quad \text { and } \quad x(s)>0 \quad \text { for all } s \in\left(t_{2}, t_{3}\right) .
$$

We will first estimate $x^{\prime}\left(t_{2}\right)$. Let $c>d_{2}$. Suppose $x^{\prime}\left(t_{2}\right)<-3 a c^{\alpha}$. Then by (2.40) and (2.43), $\operatorname{Max}\left\{\left|x^{\prime}(s)\right| ; s \in\left[t_{1}, t_{2}\right]\right\}=-x^{\prime}\left(t_{2}\right)$.

Now multiplying $(1.1)$ by $x^{\prime}(s)$ and integrating on $\left[t_{1}, t_{2}\right]$ we have

$$
\begin{aligned}
{\left[x^{\prime}\left(t_{2}\right)\right]^{2}=} & {\left[x^{\prime}\left(t_{1}\right)\right]^{2}-2 G\left(x\left(t_{2}\right)\right)+2 G\left(x\left(t_{1}\right)\right) } \\
& +2 c \int_{t_{1}}^{t_{2}} x^{\prime}(s) d s+2 \int_{t_{1}}^{t_{2}} p(s) x^{\prime}(s) d s,
\end{aligned}
$$

where $G$ is the primitive of $g$. But $x\left(t_{1}\right)=x\left(t_{2}\right)$ and so $G\left(x\left(t_{1}\right)\right)=G\left(x\left(t_{2}\right)\right)$, $\int_{t_{1}}^{t_{2}} x^{\prime}(s) d s=0$. Hence by (2.47) and (2.40) we have

$$
\left[x^{\prime}\left(t_{2}\right)\right]^{2} \leqslant 4 a^{2} c^{2 \alpha}-2 x^{\prime}\left(t_{2}\right)\left(t_{2}-t_{1}\right),
$$

that is,

$$
\left[x^{\prime}\left(t_{2}\right)\right]^{2}+2 x^{\prime}\left(t_{2}\right)\left(t_{2}-t_{1}\right)-4 a^{2} c^{2 \alpha} \leqslant 0
$$


and so

$$
x^{\prime}\left(t_{2}\right) \geqslant-\left(t_{2}-t_{1}\right)-\sqrt{\left(t_{2}-t_{1}\right)^{2}+4 a^{2} c^{2 x}} .
$$

Now by Step 2, there exists $\delta_{5} \geqslant d_{2}$ such that if $c>\delta_{5}$ then

$$
t_{2}-t_{1}<3 a c^{-\varepsilon} \text {. }
$$

Hence for such $c>\delta_{5}$ by (2.48) we get

$$
-3 a c^{\alpha}>-3 a c^{-\varepsilon}-\sqrt{9 a^{2} c^{-2 \varepsilon}+4 a^{2} c^{2 \alpha}}
$$

which is clearly false for large enough $c$, since $\varepsilon>0$. Hence there exists $\delta_{6} \geqslant \delta_{5}$ such that for $c>\delta_{6}$

$$
x^{\prime}\left(t_{2}\right) \geqslant-3 a c^{\alpha} .
$$

In the following let $c>\delta_{6}$. Then by (2.40), (2.43), and (2.50) we have

$$
\left|x^{\prime}(s)\right| \leqslant 3 a c^{\alpha} \quad \text { for all } s \in\left[t_{1}, t_{2}\right] .
$$

Hence using (2.47), (2.30), and (2.49) we get

$$
\begin{aligned}
{\left[x^{\prime}\left(t_{2}\right)\right]^{2} } & \geqslant\left(a c^{\alpha} / 2\right)^{2}-6 a c^{\alpha}\left(t_{2}-t_{1}\right) \\
& \geqslant\left(a c^{\alpha} / 2\right)^{2}-\left(6 a c^{\alpha}\right)\left(3 a c^{-\varepsilon}\right) \\
& \geqslant\left(a c^{\alpha} / 3\right)^{2}
\end{aligned}
$$

provided $c$ is large enough. Hence there exists $\delta_{7}$ such that if $c>\delta_{7}$ then

$$
x^{\prime}\left(t_{2}\right) \leqslant-\left(a c^{\alpha} / 3\right) \text {. }
$$

Let $c>\delta_{7}$. Suppose now that for all $s \in\left[t_{2}, t_{2}+c^{\beta(\alpha-1)}\right], x(t)>0$. This, (1.1), and (2.52) imply that for all $s \in\left[t_{2}, t_{2}+c^{\beta(\alpha-1)}\right]$,

$$
\begin{aligned}
x^{\prime}(s) & \leqslant-\left(a c^{\alpha} / 3\right)+\int_{t_{2}}^{s}[c+1-g(x(\tau))] d \tau \\
& \leqslant-\left(a c^{\alpha} / 3\right)+(c+1)\left(s-t_{2}\right) .
\end{aligned}
$$

Thus

$$
\begin{aligned}
0 & <x\left(t_{2}+c^{\beta(\alpha-1)}\right) \leqslant x\left(t_{2}\right)-\left(a c^{\alpha} / 3\right) c^{\beta(\alpha-1)}+(c+1)\left[c^{2 \beta(\alpha-1)} / 2\right] \\
& =g^{-1}\left(c-1+c^{\alpha+\varepsilon}\right)+\frac{c^{1+2 \beta(x-1)}}{2}+\frac{c^{2 \beta(\alpha-1)}}{2}-\frac{a}{3} c^{\alpha+\beta(x-1)} .
\end{aligned}
$$


That is,

$$
0<g^{-1}\left(c-1+c^{\alpha+\varepsilon}\right) c^{-\alpha-\beta(\alpha-1)}+\frac{c^{(\beta-1)(\alpha-1)}}{2}+\frac{c^{\beta(\alpha-1)-\alpha}}{2}-\frac{a}{3} .
$$

Now clearly $(\beta-1)(\alpha-1)<0, \beta(\alpha-1)-\alpha<0$ and

$$
\begin{aligned}
\frac{1}{\rho+1}-\alpha-\beta(\alpha-1) & =\frac{1}{\rho+1}-\alpha+\beta(1-\alpha) \\
& <\frac{1}{\rho+1}-\alpha+\left(1+\frac{1}{\rho+1}\right)(1-\alpha) \\
& =\left(\frac{\rho+3}{\rho+1}\right)-\alpha\left(\frac{2 \rho+3}{\rho+1}\right)=0
\end{aligned}
$$

since $\alpha=(\rho+3) /(2 \rho+3)$. Then by (1.4), we can find $d_{3} \geqslant \delta_{7}$ such that if $c>d_{3}$ then (2.54) is false. That is, there must exist a $t_{3} \in\left[t_{2}, t_{2}+c^{\beta(\alpha-1)}\right]$ such that $x\left(t_{3}\right)=0$ and $x(s)>0$ for all $s \in\left[t_{2}, t_{3}\right)$, which proves Step 3 .

We will now prove Lemma 2.3. Let $c>d_{3}$. From Steps $1-3$

$$
t_{3} \leqslant T+\left(c^{-\varepsilon} / 2\right)+\left[2 a c^{\alpha} /\left(c^{\alpha+\varepsilon}-2\right)\right]+c^{\beta(\alpha-1)} .
$$

Now since $\varepsilon<(1-\alpha) / 2$ and $\beta>1,-\varepsilon>\beta(\alpha-1)$. Hence there exists $d_{4} \geqslant d_{3}$ such that if $c>d_{4}$ then (2.55) gives

$$
t_{3} \leqslant T+5 a c^{-\varepsilon} \text {. }
$$

Next we estimate $\left|x^{\prime}(s)\right|$ for $s \in\left[T, t_{3}\right]$. Now integrating (1.1) on $[T, s]$ for $s \leqslant t_{1}$ we have

$$
\begin{aligned}
x^{\prime}(s) & \leqslant a c^{\alpha}+\int_{T}^{s}(c+1) d \tau \\
& \leqslant a c^{\alpha}+(c+1)\left(c^{-\varepsilon} / 2\right) .
\end{aligned}
$$

But $x^{\prime}(s)>0($ see $(2.24))$ and hence

$$
\left|x^{\prime}(s)\right| \leqslant a c^{\alpha}+(c+1)\left(c^{-\varepsilon} / 2\right)
$$

in $[T, s]$, where $s \leqslant t_{1}$. From (2.51) we already have $\left|x^{\prime}(s)\right| \leqslant 3 a c^{\alpha}$ for all $s \in\left[t_{1}, t_{2}\right]$. Now for $s \in\left[t_{2}, t_{3}\right]$ integrating (1.1) on $\left[t_{2}, s\right]$, where $s \leqslant t_{3}$, we have

$$
x^{\prime}(s)=x^{\prime}\left(t_{2}\right)+\int_{t_{2}}^{s}[c+p(\tau)-g(x(\tau))] d \tau
$$


and so

$$
x^{\prime}\left(t_{2}\right)+\left[c-1-g\left(x\left(t_{2}\right)\right)\right]\left(s-t_{2}\right) \leqslant x^{\prime}(s) \leqslant x^{\prime}\left(t_{2}\right)+(c+1)\left(s-t_{2}\right),
$$

that is, using (2.50) and (2.52) we get

$$
-3 a c^{x}-c^{x+\varepsilon} c^{\beta(x-1)} \leqslant x^{\prime}(s) \leqslant-a c^{\alpha} / 3+(c+1) c^{\beta(x-1)} .
$$

Hence

$$
-3 a-c^{\varepsilon+\beta(\alpha-1)} \leqslant \frac{x^{\prime}(s)}{c^{\alpha}} \leqslant-\frac{a}{3}+(c+1) c^{-\alpha+\beta(\alpha-1)} .
$$

But $\varepsilon+\beta(\alpha-1)<(1-\alpha) / 2+\beta(\alpha-1)<0$ since $\beta>1$ and $1-\alpha+\beta(\alpha-1)$ $=(\alpha-1)(\beta-1)<0$ since $\alpha<1, \beta>1$. Then there exists $d_{5} \geqslant d_{4}$ such that if $c>d_{5}$ (see (2.58)) we have

$$
-4 a c^{\alpha} \leqslant x^{\prime}(s) \leqslant-(a / 4) c^{\alpha},
$$

for $s \in\left[t_{2}, t_{3}\right]$. Hence combining (2.59), (2.51), and (2.57), for $c>d_{5}$ we have

$$
\left|x^{\prime}(s)\right| \leqslant \operatorname{Max}\left\{a c^{\alpha}+(c+1) c^{-\varepsilon} / 2,4 a c^{\alpha}\right\} \quad \text { for } \quad s \in\left[T, t_{3}\right] .
$$

But $1-\varepsilon>\alpha$. Hence there exists $d_{6} \geqslant d_{5}$ such that if $c>d_{6}$ then for $s \in\left[T, t_{3}\right]$ we have

$$
\left|x^{\prime}(s)\right| \leqslant c^{1-\varepsilon} .
$$

Now multiplying (1.1) by $x^{\prime}(s)$ and integrating on $\left[T, t_{3}\right]$, using the fact that $x\left(t_{3}\right)=x(T)=0$, we get

$$
x^{\prime}\left(t_{3}\right)^{2}-x^{\prime}(T)^{2}=2 \int_{T}^{t_{3}} p(s) x^{\prime}(s) d s .
$$

Hence by using (2.61), (2.56), and the fact that $x^{\prime}(T)=a c^{\alpha}$ we have

$$
\left|x^{\prime}\left(t_{3}\right)^{2}-a^{2} c^{2 \alpha}\right| \leqslant 10 a c^{1-2 \varepsilon}
$$

Now (2.63) gives

$$
\left|x^{\prime}\left(t_{3}\right)+a c^{\alpha}\right| \leqslant \frac{10 a c^{t-2 \varepsilon}}{\left|x^{\prime}\left(t_{3}\right)-a c^{\alpha}\right|}
$$

and since $x^{\prime}\left(t_{3}\right)<0($ see $(2.59))$ we get

$$
\left|x^{\prime}\left(t_{3}\right)+a c^{\alpha}\right| \leqslant 10 c^{1-\alpha-2 \varepsilon} \leqslant c^{\alpha}\left[10 c^{1-2 x-2 \varepsilon}\right] .
$$


But $1-2 \alpha-2 \varepsilon<0$ since $2 \alpha=(2 \rho+6) /(2 \rho+3)>1$ and $\varepsilon>0$. Hence there exists $d(n) \geqslant d_{6}$ such that if $c>d=d(n)$

$$
10 c^{1-2 \alpha-2 \varepsilon}<1 /\left(2 n^{2}\right),
$$

and

$$
5 a c^{-\varepsilon}<1 / n
$$

Now for $c>d$, combining (2.56) and (2.66) we get

$$
t_{3} \leqslant T+1 / n,
$$

and combining (2.65) and (2.64) we get

$$
\left|x^{\prime}\left(t_{3}\right)+a c^{\alpha}\right|<c^{\alpha} /\left(2 n^{2}\right) .
$$

Hence clearly $T_{1}=t_{3}-T$ satisfies the requirements of Lemma 2.3. Thus Lemma 2.3 is proven.

LEMMA 2.4. Let $x(t)$ be a solution to (1.1) satisfying $x(T)=0$, $x^{\prime}(T)=-a c^{\alpha}$ with $(T, a) \in[0,1] \times[1,3]$. Given any positive integer $n$ there exists $D:=D(N)$ such that if $c>D$ then there exists $T_{1} \in(0,1 / n]$ with $x\left(T+T_{1}\right)=0$ and $x<0$ on $\left(T, T+T_{1}\right)$. Moreover

$$
\left|x^{\prime}\left(T+T_{1}\right)-a c^{\alpha}\right|<c^{\alpha} /\left(2 n^{2}\right) \text {. }
$$

Proof. Let $0<\tau<1$ be such that $M-\tau \neq 0$. By (1.3) there exists a real number $\eta$ such that

$$
(M-\tau) x-g(x) \geqslant \eta \quad \text { for all } x \leqslant 0 .
$$

Now we define

$$
f(u):= \begin{cases}\left(\sinh \left((\tau-M)^{1 / 2}(u-T)\right)\right) /(\tau-M)^{1 / 2} & \text { if } \tau>M \\ \left(\sin \left((M-\tau)^{1 / 2}(u-T)\right)\right) /(M-\tau)^{1 / 2} & \text { if } \tau<M .\end{cases}
$$

Then rewriting $(1.1)$ as $x^{\prime \prime}+(M-\tau) x=c+p(t)+(M-\tau) x-g(x(t))$ we have

$$
x(t)=-a c^{\alpha} f(t)+\int_{T}^{t} f(T+t-s)(p(s)+c+(M-\tau) x(s)-g(x(s))) d s .
$$

Now consider the interval $\left[T, T+c^{(\alpha-1) / 4}\right]$. Since $0<\alpha<1$, there exists $D_{1}(n)$ such that if $c>D_{1}$

$$
c^{(\alpha-1) / 4} \leqslant \pi /\left(2|\tau-M|^{1 / 2}\right) .
$$


Let $c>D_{1}$. Then in $\left[T, T+c^{(\alpha} 1^{1) / 4}\right], f \geqslant 0$ and hence from $(2.70)-(2.72)$ we have

$$
x(t) \geqslant-a c^{\alpha} f(t)+(c+\eta-1) \int_{T}^{t} f(T+t-s) d s .
$$

But in $\left[T, T+c^{(x-1) / 4}\right]$ we also have

$$
2(t-T) / \pi \leqslant f(t) \leqslant\left(e^{\pi / 2}\right) /\left(2|\tau-M|^{1 / 2}\right)=K_{1} \quad \text { (say). }
$$

Now let $c>D_{2}$, where $D_{2} \geqslant D_{1}$ is such that $c+\eta-1 \geqslant c / 2$. Then from (2.74) and (2.75) we get

$$
x(t) \geqslant-a c^{\alpha} K_{1}+(c / 2)(t-T)^{2} / \pi
$$

for all $t \in\left[T, T+c^{(\alpha-1) / 4}\right]$. In particular,

$$
\begin{aligned}
x\left(T+c^{(x-1) / 4}\right) & \geqslant-a c^{\alpha} K_{1}+\left[c c^{(x-1) / 2}\right] /(2 \pi) \\
& =-a c^{\alpha} K_{1}+c^{(x+1) / 2} /(2 \pi) .
\end{aligned}
$$

But $(\alpha+1) / 2>\alpha$. So there exists $D_{3} \geqslant D_{2}$ such that if $c>D_{3}$ then $x\left(T+c^{(x-1) / 4}\right)>0$. Hence choosing $c \geqslant D_{4}=D_{4}(n)$, where $D_{4} \geqslant D_{3}$ is such that

$$
c^{(x-1) / 4}<1 / n
$$

there exists $T_{1} \in[0,1 / n]$ such that $x\left(T+T_{1}\right)=0$ and $x(s)<0$ on $\left(T, T+T_{1}\right)$.

We conclude the proof of Lemma 2.4 by establishing (2.69). From (2.76) we have that $x(t) \geqslant-K_{1} a c^{\alpha}$ for all $t \in\left[T, T+T_{1}\right]$. Then using (1.3), there exists $K_{2}>0, D_{5}$, where $D_{5} \geqslant D_{4}$ such that for $c>D_{5}$

$$
\left|x^{\prime \prime}(t)\right| \leqslant K_{2} c
$$

for all $t \in\left[T, T+T_{1}\right]$ and so

$$
\left|x^{\prime}(t)-x^{\prime}(T)\right| \leqslant K_{2} c(t-T)
$$

for all $t \in\left[T, T+T_{1}\right]$. But $T_{1}<c^{(\alpha-1) / 4}$ since $x\left(T+c^{(\alpha-1) / 4}\right)>0$. Hence

$$
\left|x^{\prime}(t)-x^{\prime}(T)\right| \leqslant K_{2} c^{1-(1-x) / 4},
$$

that is,

$$
-a c^{\alpha}-K_{2} c^{1 \cdots \varepsilon_{1}} \leqslant x^{\prime}(t) \leqslant-a c^{\alpha}+K_{2} c^{1-\varepsilon_{1}}
$$


for all $t \in\left[T, T+T_{1}\right]$, where

$$
\varepsilon_{1}=(1-\alpha) / 4
$$

Now clearly $1-\varepsilon_{1}>\alpha$. Hence there exists $K_{3}>0, D_{6}$, where $D_{6} \geqslant D_{5}$ such that for $c>D_{6}$

$$
\left|x^{\prime}(t)\right| \leqslant K_{3} c^{1-\varepsilon_{1}}
$$

for all $t \in\left[T, T+T_{1}\right]$. The rest of the proof of (2.69) follows the same pattern of proving (2.15). For this reason we refer the reader to $(2.62)-(2.68)$. This concludes the proof of Lemma 2.4 .

Lemma 2.5. Let $m>0, \delta>0$ be such that $M+m>0$. Let $x(t)$ be $a$ solution to (1.1) and let $c^{*}$ be as in Lemma 2.2. For each $c>c^{*}$ there exists $B:=B(c, m, \delta)>0$ such that if $b>B$, if $x\left(t_{1}\right)=0$, and if $x^{\prime}\left(t_{1}\right)=-b$ for some $t_{1} \in[0,1]$, then $x(t)<0$ for all $t \in\left(t_{1}, t_{1}+\pi(M+m+\delta)^{-1 / 2}\right)$.

Proof. By (1.3)-(1.4) we see that there exists a constant $M^{*}<0$ such that

$$
g(u)-(M+m) u>M^{*}
$$

for all $u \in R$. Let $\lambda=(M+m)^{1 / 2}$. Rewriting (1.1) as $x^{\prime \prime}(s)+\lambda^{2} x(s)=$ $c+p(s)-g(x(s))+\lambda^{2} x(s)$ we have for $s \in(0, \pi / \lambda)$

$$
\begin{aligned}
x\left(t_{1}+s\right)= & \lambda^{-1}\left\{x^{\prime}\left(t_{1}\right) \sin (\lambda s)+\int_{0}^{s}\left[\operatorname { s i n } ( \lambda ( s - u ) ) \left(c+p\left(t_{1}+u\right)\right.\right.\right. \\
& \left.\left.\left.-g\left(x\left(t_{1}+u\right)\right)+\lambda^{2}\left(x\left(t_{1}+u\right)\right)\right)\right] d u\right\} \\
\leqslant & \lambda^{-1} \sin (\lambda s)\left\{-b+\frac{\left(c+1-M^{*}\right)[1-\cos (\lambda s)]}{\lambda \sin (\lambda s)}\right\} \\
= & \lambda^{-1} \sin (\lambda s)\left\{-b+\frac{\left(c+1-M^{*}\right)}{\lambda} \tan (\lambda s / 2)\right\}
\end{aligned}
$$

where we have used (2.84). From (2.85) it follows immediately that if

$$
\begin{aligned}
& b>\left[\left(c+1-M^{*}\right) /(M+m)^{1 / 2}\right] \tan \left([M+m]^{1 / 2} \pi /\left[2(M+m+\delta)^{1 / 2}\right]\right) \\
& \quad:=B(c, m, \delta)
\end{aligned}
$$

then $x(t)<0$ for all $t \in\left(t_{1}, t_{1}+\pi(M+m+\delta)^{-1 / 2}\right)$, and Lemma 2.5 is proven. 
Lemma 2.6. Let $c>c^{*}$ be given and $B(c, m, \delta)$ be as above. We claim that there exists $F:=F(c, m, \delta)>0$ such that if $x(t)$ satisfies $(1.1), x(0)=0$, $x^{\prime}(0)=b$, where $|b|>F$, then $\left|x^{\prime}(T)\right|>B(c, m, \delta)$ whenever $x(T)=0$, $0 \leqslant T \leqslant 1$.

Proof. Let $E_{1}$ be as in Lemma 2.1. By a similar argument as in (2.3) (2.4) we obtain

$$
\left(d E_{1} / d t\right) \geqslant-\sqrt{2}|c+1|-\left(|c+1|+M_{1} \sqrt{2}\right) E_{1} .
$$

Multiplying (2.87) by $e^{k t}$, where $k=|c+1|+M_{1} \sqrt{2}$, we have

$$
\frac{d}{d t}\left(e^{k t} E_{1}\right) \geqslant-\sqrt{2}|c+1| e^{k t}
$$

and so integrating on $[0, T]$ we have

$$
e^{k T} E_{1}(T)-E_{1}(0) \geqslant-(\sqrt{2} / k)|c+1|\left\{e^{k T}-1\right\},
$$

and since $E_{1}(0)=b^{2} / 2$ we obtain

$$
\begin{aligned}
E_{1}(T) & \geqslant e^{-k T}\left\{\left(b^{2} / 2\right)+\frac{\sqrt{2}|c+1|}{k}\right\}-\frac{\sqrt{2}|c+1|}{k} \\
& \geqslant e^{-k}\left(b^{2} / 2\right)-(\sqrt{2}|c+1| / k) .
\end{aligned}
$$

But $E_{1}(T)=\left[x^{\prime}(T)\right]^{2} / 2$. Hence from $(2.88)$ we have $\left|x^{\prime}(T)\right|=\left(2 E_{1}(T)\right)^{1 / 2}>$ $B(c, m, \delta)$ whenever

$$
\begin{aligned}
\left|x^{\prime}(0)\right| & =|b|>\left\{\left[B^{2}(c, m, \delta)+2 \sqrt{2}|c+1| k\right] e^{k}\right\}^{1 / 2} \\
& :=F(c, m, \delta),
\end{aligned}
$$

which proves Lemma 2.6 .

\section{Proof of Theorem 1.1}

Let $n>\left[2\left(\operatorname{Max}\{0, M\}^{1 / 2}\right) / \pi\right]$ be an integer. Let $c>\max \{d(n+1)$, $\left.D(n+1), c^{*}\right\}$ (see Lemmas 2.2-2.6). From (2.12) it follows immediately that if $\theta\left(t_{1}, a\right)=\pi K$ with $K$ a non-negative integer, then

$$
\theta(t, a)>\pi K \quad \text { for all } t>t_{1} .
$$

First we will let $n>\left[2\left(\operatorname{Max}\{0, M\}^{1 / 2}\right) / \pi\right]+1$ be an integer and establish a solution $x(t)$ to $(1.1)-(1.2)$ with $n$ interior zeroes and satisfying $x^{\prime}(0)>0$. 
Since $\theta(0,2)=0$, by Lemma 2.3 there exists $T_{1} \in(0,1 /(n+1)]$ such that $\theta\left(T_{1}, 2\right)=\pi$ and by $(2.15)$ we see that

$$
x^{\prime}\left(T_{1}, 2\right) \in\left[-\left(2+1 /\left(2(n+1)^{2}\right)\right) c^{\alpha},-\left(2-1 /\left(2(n+1)^{2}\right)\right) c^{\alpha}\right] .
$$

Hence by Lemma 2.4 we see that there exists $T_{2} \in\left(T_{1}, 2 /(n+1)\right]$ such that $\theta\left(T_{2}, 2\right)=2 \pi$ and

$$
\left|x^{\prime}\left(T_{2}, 2\right)+x^{\prime}\left(T_{1}, 2\right)\right| \leqslant c^{\alpha} /\left(2(n+1)^{2}\right) .
$$

Then (3.1) yields

$$
\theta(2 /(n+1), 2) \geqslant 2 \pi,
$$

and from (3.2), (3.3) we have

$$
\begin{aligned}
\left|x^{\prime}\left(T_{2}, 2\right)-2 c^{\alpha}\right| \leqslant & \left|x^{\prime}\left(T_{2}, 2\right)+x^{\prime}\left(T_{1}, 2\right)\right| \\
& +\left|x^{\prime}\left(T_{1}, 2\right)+2 c^{\alpha}\right| \\
\leqslant & c^{\alpha} /\left(2(n+1)^{2}\right)+c^{\alpha} /\left(2(n+1)^{2}\right)=c^{\alpha} /(n+1)^{2} .
\end{aligned}
$$

Itcrating this argument (i.c., applying Lemmas 2.3 and 2.4 consecutively $(n+1) / 2$ times $)$ we obtain

$$
\theta(1,2)>(n+1) \pi \text {. }
$$

Let now $m>0$ and $\delta>0$ be such that $M+m>0$ and $n>$ $\left[2(M+m+\delta)^{1 / 2} / \pi\right]+1$. Let $b>\max \left\{2 c^{\alpha}, F(c, m, \delta)\right\}$, where $F(c, m, \delta)$ is as in Lemma 2.6 and consider $x(t)$ satisfying $(1.1), x(0)=0, x^{\prime}(0)=b$. Since $c>c^{*}, b>c^{\alpha}$ by Lemma 2.2 there exists a non-negative integer $I$ such that $I \pi \leqslant \theta\left(1, b c^{-x}\right)<(I+1) \pi$. Further by (3.1) there exists an increasing sequence $0=t_{0}<t_{1}<t_{2}<\cdots<t_{1} \leqslant 1$ such that

$$
\theta\left(t_{i}, b c^{-\alpha}\right)=\pi i, \quad i=0,1,2, \ldots, I .
$$

Now since $b>F(c, m, \delta)$, by Lemmas 2.5 and 2.6 we obtain that

$$
\left|x^{\prime}\left(t_{i}\right)\right| \geqslant B(c, m, \delta), \quad i=1,2, \ldots, I
$$

and

$$
t_{2 j}-t_{2 j-1} \geqslant \pi(M+m+\delta)^{-1 / 2} .
$$

Since $t_{I} \leqslant 1$, from (3.9) we infer

$$
\begin{aligned}
1 & \geqslant t_{I}=\sum_{i=1}^{I} t_{i}-t_{i-1} \geqslant \sum_{j=1}^{[[I / 2]]} t_{2 j}-t_{2 j-1} \\
& \geqslant[[I / 2]] \pi(M+m+\delta)^{-1 / 2} .
\end{aligned}
$$


Thus

$$
\begin{aligned}
\theta\left(1, b c^{-x}\right) & \leqslant(I+1) \pi \leqslant(2[[I / 2]]+2) \pi \\
& \leqslant 2(M+m+\delta)^{1 / 2}+2 \pi<(n+1) \pi .
\end{aligned}
$$

From (3.6), (3.11), the continuous dependence of $\theta(1, a)$ on $\mathbf{a}$, and the intermediate value theorem we see that there exists $a_{1} \in\left[2, b c^{-x}\right]$ such that

$$
\theta\left(1, a_{1}\right)=(n+1) \pi
$$

By the definition of $\theta$ it follows then that $x\left(t, a_{1}\right)$ is a solution of $(1.1)-(1.2)$. In addition by $(3.1)$ it follows that $x\left(t, a_{1}\right)$ has exactly $n$ interior zeroes, and the $(\mathrm{A})$ part is proven.

Next we let $n>\left[2\left(\operatorname{Max}\{0, M\}^{1 / 2}\right) / \pi\right]$ be an integer and obtain a second solution to (1.1)-(1.2), $x_{1}(t)$, having $n$ interior zeroes. Unlike our first solution, $x_{1}(t)$ satisfies

$$
x_{1}^{\prime}(0)<0 .
$$

Let $x(t, a)$ denote the solution to (1.1) satisfying $x(0)=0, x^{\prime}(0)=a c^{\alpha}$ with $a \in(-\infty,-1]$. Then we can define a new angle function $\psi(t, a)$ by

$$
\begin{gathered}
x(t, a)=-r(t, a) \sin (\psi(t, a)) \\
x^{\prime}(t, a)=-r(t, a) \cos (\psi(t, a)),
\end{gathered}
$$

as long as $r(t, a)>0$. Thus by Lemma $2.2, \psi$ is well defined on $[0,2] \times$ $(-\infty,-1]$ if $c>c^{*}$. Since we are assuming $c>D(n+1)$, by Lemma 2.4 there exists $\tau_{1} \in(0,1 /(n+1)]$ such that

$$
\psi\left(\tau_{1},-2\right)=\pi, \quad\left|x^{\prime}\left(\tau_{1},-2\right)-2 c^{\alpha}\right|<c^{\alpha} /\left(2(n+1)^{2}\right) .
$$

Applying now Lemma 2.3 we see that there exists $\tau_{2} \in\left(\tau_{1}, 2 /(n+1)\right]$ such that

$$
\psi\left(\tau_{2},-2\right)=2 \pi, \quad\left|x^{\prime}\left(\tau_{2},-2\right)+x^{\prime}\left(\tau_{1},-2\right)\right|<c^{\alpha} /\left(2(n+1)^{2}\right),
$$

and so

$$
\begin{aligned}
\left|x^{\prime}\left(\tau_{2},-2\right)+2 c^{\alpha}\right| & \leqslant\left|x^{\prime}\left(\tau_{2},-2\right)+x^{\prime}\left(\tau_{1},-2\right)\right|+\left|2 c^{\alpha}-x^{\prime}\left(\tau_{1},-2\right)\right| \\
& \leqslant c^{\alpha} /\left(2(n+1)^{2}\right)+c^{\alpha} /\left(2(n+1)^{2}\right) \\
& =c^{\alpha} /(n+1)^{2} .
\end{aligned}
$$

Iterating this process and using (3.1) we have

$$
\psi(1,-2) \geqslant(n+1) \pi
$$


Let now $m>0$ and $\delta>0$ be such that $M+m>0$ and $n>2(M+m+\delta)^{1 / 2} / \pi$. Let $b<0$ be such that $|b|>\max \left\{2 c^{\alpha}, F(c, m, \delta)\right\}$, where $F(c, m, \delta)$ is as in Lemma 2.6, and consider $x(t)$ satisfying (1.1), $x(0)=0, x^{\prime}(0)=b$. Since $c>c^{*},|b|>c^{\alpha}$, by Lemma 2.2 there exists a nonnegative integer $K$ such that $K \pi \leqslant \psi\left(1, b c^{-\alpha}\right)<(K+1) \pi$.

Further by (3.1) there exists an increasing sequence $0=\tau_{0}<\tau_{1}<\tau_{2}<$ $\cdots<\tau_{K} \leqslant 1$ such that

$$
\psi\left(\tau_{i}, b c^{-\alpha}\right)=\pi i, \quad i=0,1,2, \ldots, K .
$$

Now since $|b|>F(c, m, \delta)$, by Lemmas 2.5 and 2.6 we obtain that

$$
\left|x^{\prime}\left(\tau_{i}\right)\right| \geqslant B(c, m, \delta), \quad i=0,1,2, \ldots, K
$$

and

$$
\tau_{2 j-1}-\tau_{2 j-2} \geqslant \pi(M+m+\delta)^{-1 / 2}
$$

Since $\tau_{K} \leqslant 1$, from (3.22) we infer

$$
\begin{aligned}
1 \geqslant \tau_{K}=\sum_{i=1}^{K} \tau_{i}-\tau_{i-1} & \geqslant \sum_{j=1}^{[[(K+1) / 2]]}\left(\tau_{2 j-1}-\tau_{2 j-2}\right) \\
& \geqslant[[(K+1) / 2]] \pi(M+m+\delta)^{-1 / 2}
\end{aligned}
$$

Thus

$$
\begin{aligned}
\psi\left(1, b c^{-\alpha}\right) & \leqslant(K+1) \pi \leqslant(2[[(K+1) / 2]]+1) \pi \\
& \leqslant 2(M+m+\delta)^{1 / 2}+\pi<(n+1) \pi
\end{aligned}
$$

From (3.19), (3.24), the continuous dependence of $\psi(1, a)$ on $\mathbf{a}$, and the intermediate value theorem we see that there exists $b_{1} \in\left[b c^{-\alpha},-2\right]$ such that

$$
\psi\left(1, b_{1}\right)=(n+1) \pi
$$

By the definition of $\psi$ it follows then that $x_{1}(t)=x\left(t, b_{1}\right)$ is a solution of (1.1) $-(1.2)$, which has exactly $n$ interior zeroes and $x_{1}^{\prime}(0)<0$. Hence the (B) part is proven.

In order to conclude the proof of Theorem 1.1 we need to show the existence of $c_{*}$. Since $g(u)-\pi^{2} u$ is bounded below, there exists a real number $J$ such that

$$
g(u)-\pi^{2} u>J
$$


for all $u \in R$. Thus, if $x(t)$ is a soluton to (1.1)-(1.2) then multiplying (1.1) by $\sin (\pi t)$ and integrating by parts we have

$$
\begin{aligned}
c \int_{0}^{1} \sin (\pi t) d t & =\int_{0}^{1}\left[g(x(t))-\pi^{2} x(t)-p(t)\right] \sin (\pi t) d t \\
& \geqslant \int_{0}^{1}[J-p(t)] \sin (\pi t) d t .
\end{aligned}
$$

This shows that (1.1)-(1.2) has no solution if

$$
c<c_{*}=(\pi / 2)\left(\int_{0}^{t}[J-p(t)] \sin (\pi t) d t\right),
$$

which concludes the proof of Theorem 1.1.

Remark. While studying the case $p(t) \equiv 0$ in [3], we established two theorems related to our work here, one if $M<\sqrt{2} \pi^{2}$ and the other if $M \geqslant \sqrt{2} \pi^{2}$. However, we recently noticed (our thanks to Mr. Terry McCabe) that the above inequalities in [3] should be corrected to $M<\pi^{2}$ and $M \geqslant \pi^{2}$, respectively, for the two theorems to hold. This can be easily seen, since for $M \geqslant 0, \lim _{q \rightarrow-\infty}[-J(q)]$ is equal to $\pi / \sqrt{2 M}$ and not $\pi^{2} / M$ as stated in Eq. (3.2) in [3].

\section{REFERENCES}

1. A. Ambrosetti and G. Prodi, On the inversion of some differentiable mappings with singularities between Banach spaces, Ann. Mat. Pura Appl. 93 (1972), 213-246.

2. A. CAStro and A. C. LazFr, On periodic solutions of weakly coupled systems of differential equations, Boll. Un. Mat. Ital. B (5) 18 (1981), 733-742.

3. A. Castro and R. Shivajl, Multiple solutions for a Dirichlet problem with jumping nonlinearities, in "Trends in Theory and Practice of Nonlinear Analysis" (V. Lakshmikantham, Ed.), North-Holland, Amsterdam, 1985.

4. E. A. Coddington and N. Levinson, "Theory of Ordinary Differential Equations," McGraw-Hill, New York, 1955.

5. A. C. LAzer and P. J. MCKenNa, On a conjecture related to the number of solutions of a nonlinear Dirichlet problem, Proc. Roy. Soc. Edinburgh Sect. A 95 (1983), 275-283. 